\title{
Planejamento estadual no SUS: \\ o caso da Secretaria da Saúde do Estado da Bahia
}

\author{
Planning in Brazilian Public Health System: \\ the case of the Health Secretariat of State of Bahia
}

Washington Luiz Abreu de Jesus ${ }^{1}$

Carmen Fontes Teixeira ${ }^{2}$

\footnotetext{
${ }^{1}$ Departamento deSaúdeda Universidade Estadual de Feira deSantana. Av. Transnordestina s/n, Novo H orizonte. 44.036-900

Feira deSantana BA.

washingtonluiz.abreudejesus @gmail.com

${ }^{2}$ Instituto de Humanidades, Artes e Ciências Prof.

Milton Santos,

UniversidadeFederal da

Bahia.
}

Abstract Theobject of this article is the planning process of the H ealth Secretariat of State of Bahia from January 2007 to July 2009. It describes and analysesthis process, discussing the difficultiesand the advances to build the planning practice in this institution. It was used documental analyses and systematic registers of lived experience by the authors to do it. The process points for an approach between theory and practice articulating the Strategic-situational focus and the Communicative Action, including the principle of the Social Participation. The analyses explain that the process occurs in a pool of dialogic movements enlaced by the explicative, normative, strategic and tactic-operative moments that finished with the construction of the H ealth Secretariat of State of Bahia. There is a powerful planning revealed in plurality and multiplicity of approaches used to build a collective "vision of future" in organizations that adopted "plans" like instruments to qualify Government actions.

Key words Public Health, Health planning, Governmental planning, Plans and government programs, Brazilian public health system
Resumo Esteartigo toma como objeto o processo deplanejamento da Secretaria da Saúde do Estado da Bahia no período compreendido entre janeiro de 2007 ejulho de 2009. Busca-se descrever eanalisar esse processo, discutindo-se algumas dificuldades eavanços no aperfeiçoamento da prática de planejamento em saúde, por meio de uma análise de documentos desenvol vidos no período pela instituição e de registros sistemáticos dos autores sobre a experiência vivenciada. 0 processo se desenvolvenuma tentativa de aproximar a teoria à prática, com a articulação do enfoque estratégico-situacional à perspectiva do agir comunicativo, tendo como princípio fundamental a participação social. A análise demonstra um conjunto demovimentos dialógicos, articulados por meio dos momentos explicativo, normativo, estratégico e tático-operacional, que culminam com a formulação do Plano Estadual deSaúde. Revela também a potência do planejamento diante da pluralidade e multiplicidadedeabordagensutilizadas para construir col etivamente uma visão de futuro em instituições queadotam "planos" como sendo apostasà qualificação das ações de governo.

Pal avras-chave Saúde pública, Planejamento em saúde, Planejamento governamental, Planoseprogramas de governo, Sistema Ú nico de Saúde 
Introdução

0 debate sobre planejamento e gestão de sistemas de saúde, ocorrido no contexto da reforma sanitária brasileira, vem inspirando o desenvolvimento deestudos quetratam da institucionalização de práticas de planejamento entendido como "ferramenta de governo", "tecnologia de gestão de sistemas de saúde" ou "instrumento de mudança na organização do processo de trabaIho em saúde" ${ }^{1-3}$.

$\mathrm{Na}$ Bahia, o planejamento foi incorporado na Secretaria de Estado da Saúde (SESAB Saúde) desde a década de setenta ${ }^{4}$, com 0 desenvolvimento dos programas de extensão de cobertura e, de modo mais intensivo, a partir do ano de $1987^{5}$, quando, no contexto de implantação do Sistema Unificado eDescentralizado deSaúde(SUDS), foi elaborado o primeiro Plano Estadual de Saúde.

Ao longo da década de noventa ${ }^{6}$ ena primeira metade da década de $2000^{7}$, os processos de planejamento na instituição permitiram a elaboração e implementação de quatro planos de saúde, além de outros instrumentos de planejamento, 0 que permitiu ao estado avançar, em 2004, para a Gestão Plena do Sistema Estadual de Saúde.

A partir de janeiro de 2007, com a mudança do governo estadual, a SESAB deu início a uma nova etapa no processo de construção do SUS, buscando fortalecer a gestão das políticas de saúde no âmbito estadual e municipal. N esse contexto, tratou-se de promover a articulação entre os vários momentos e instrumentos do planejamento governamental e em saúde em particular, apoiando-se na iniciativa do Sistema de Planejamento do SUS ${ }^{8}$, conduzida em âmbito nacional pelo M inistério da Saúde.

O planejamento, desse modo, vem se constituindo em um espaço de inovação metodológica e organizacional, contemplando a incorporação e experimentação de técnicas e instrumentos oriundos de diversas correntes de pensamento na área. Essa experiência vem sendo sistematizada pela equipe de condução do processo e por parceiros institucionais envolvidos por meio de cooperação técnica como a Organização PanAmericana de Saúde (OPAS/O MS) e a Universidade Federal da Bahia (UFBA).

Considerando estudos recentes sobre o "estado da arte" do planejamento e gestão em saúde $^{9,10}$ e o levantamento bibliográfico realizado para fundamentar este trabalho, por meio das bases de dados SciELO e LILACS nos últimos cinco anos, pode-se observar a rel evância e atualidade de pesquisas que abordam o processo de institucionalização de práticas de planejamento em saúde no âmbito estadual e municipal11-15.

Nessa perspectiva, o objetivo desse trabalho é descrever e analisar o processo de planejamento desenvolvido naSESAB, buscando discutir algumas das dificuldades encontradas e os avanços no aperfeiçoamento da prática de planejamento identificados, no período de janeiro de 2007 a julho de 2009.

Para isso, realizamos a identificação, seleção e codificação de documentos produzidos bem como os resumos executivos das reuniões, encontros e oficinas realizadas no processo de planejamento, cuja leitura sistemática teve como instrumento de apoio o caderno de registros de um dos autores, construído na perspectiva de uma pesquisa em processo ${ }^{16}$. Buscou-se descrever e analisar as atividades desenvolvidas, destacando o esforço de articulação da prática de planejamento e dos conteúdos dos planos elaborados, especialmente o Plano Estadual de Saúde (PES 2008-2011) ${ }^{17}$, o plano plurianual (PPA) ${ }^{18}$ ea agenda estratégica da SESAB (2007-2010) ${ }^{19-21}$.

\section{Marco teórico-conceitual}

O processo de planejamento na SESAB, entendida como uma organização "hipercomplexa"22,23, busca aproximar teoriae prática, num movimento que utiliza o enfoque estratégico-situacional $(\mathrm{PES})^{24-27}$, articulado à perspectiva do agir comunicativo em Saúde ${ }^{28,29}$ eda organização queapren$\mathrm{de}^{29,30}$, considerando a participação social ${ }^{31}$ como princípio fundamental na construção das políticas públicas - característica importante de processos democráticos de governo.

De acordo com Matus, o planejamento estratégico-situacional ${ }^{25}$ é constituído por quatro momentos em constante interação, forma complexa e apropriada de entender a dinâmica dos processos de planejamento. Esses momentos encadeiam-se e formam circuitos repetitivos para ajudarem-se mutuamente, podendo ser aplicados concomitantemente para uma grande diversidade de problemas e oportunidades ${ }^{26,27}$.

No momento explicativo, ocorre a seleção e explicação dos problemas. 0 momento normativo constitui-se em uma instância de desenho dos enfrentamentos aos problemas identificados, em que se deve centrar a aten ção na eficácia das operações para alcançar a situação-objetivo. No momento estratégico, discutem-se as possíveis restrições ao cumprimento do desenho normativo, considerando os recursos de poder político 
e econômico, bem como as capacidades organizacionais einstitucionais. E no momento táticooperacional, interfere-se sobre a realidade e se avalia as ações realizadas ${ }^{25-27}$.

Além da organização dos momentos do planejamento, adotou-se a abordagem comunicativa do PES proposta por Rivera e Artmann ${ }^{28}$, Cujos postulados pautam-se no reconhecimento da negociação, liderança ecultura institucional como parte do processo de gestão. Os autores propõem uma ampliação do enfoque dado pelo planejamento estratégico-situacional, considerando a perspectiva da "ação comunicativa" como fundamental para a construção de organizações dialógicas capazes de construir-se a partir da ação dos sujeitos sobre projetos institucionais coletivos.

N essa perspectiva, buscou-se valorizar os diferentes atores sociais em situação, considerando-se necessário adotar também a perspectiva da "organização que aprende" 28,29 , na qual se reconhece, que para construir projetos coletivos, é preciso estabelecer pensamento sistêmico, promover a aprendizagem em equipeeconstruir uma visão compartilhada por meio do diálogo com os diferentes modelos mentais que compõem 0 ambienteinstitucional.

O processo deelaboração do Plano Estadual de Saúde 2008-2011

O Plano Estadual de Saúde 2008-2011 foi construído a partir de um conjunto de processos ar- ticulados e interdependentes, iniciados desde os primeiros dias da gestão atual, os quais podem ser sistematizados em sete movimentos.

\section{Primeiro movimento:}

a proposta do "planejamento integrado"

Um dos desafios colocados para o planejamento no contexto da nova gestão foi articular os diversos instrumentos de planejamento de modo a conferir maior coerência entre eles, buscando a convergência de proposições, métodose formas de organização dos processos de trabaIho. Nessa perspectiva, a Assessoria de Planejamento ao Gabinete do Secretário da Saúde (GA$\mathrm{SEC})$, constituída por uma pequena equipe técnica, se encarregou de organizar um processo de planejamento queintegra os diversos instrumentos de gestão do SUS-Bahia, articulando-os por meio das conexões conceituais. M antêm- secomo elementos centrais dessa articulação os problemas, demandas e oportunidades, que, no desdobramento, são enfrentados pelas diversas linhas de ação que se constituem na espinha dorsal de todo o processo, como se demonstrará adiante, conforme explicitado na Figura 1.

Segundo movimento: definição das "linhas de ação" de gover no e construção dos instrumentos estratégicos da gestão

No processo de planejamento institucional, o percurso incluiu a definição das"linhas deação"

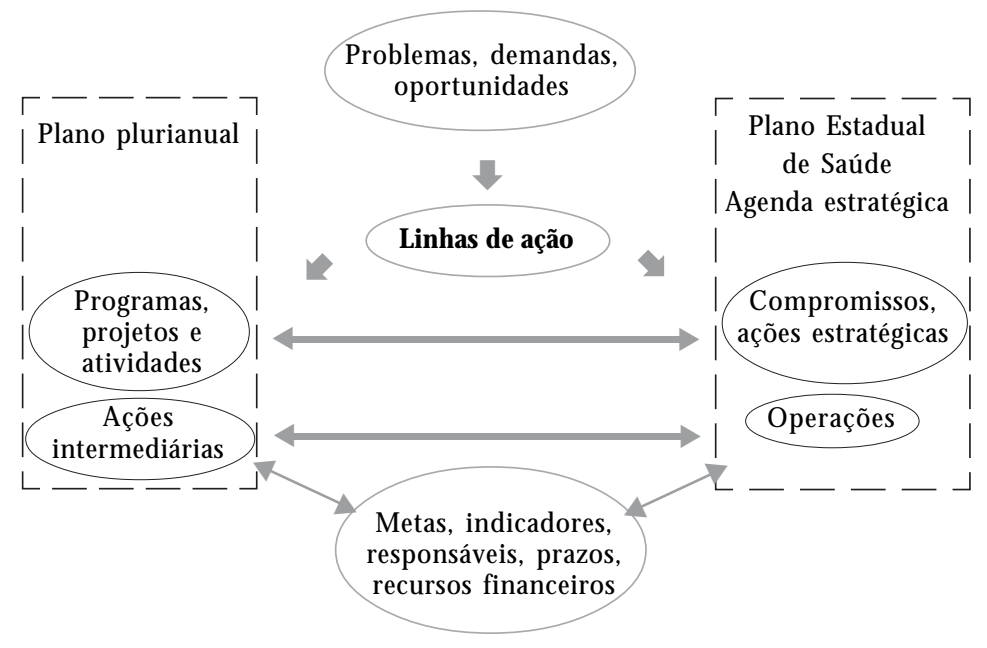

Figura 1. Quadro de conexões conceituais do planejamento na SESAB. Bahia, 2007. 
para 0 alcance dos objetivos estabelecidos pelo Colegiado de Gestão, o que ocorreu entre os meses de janeiro e dezembro de 2007: gestão de mocrática, solidária e efetiva do SUS, gestão do trabalho e da educação permanente em saúde, vigilância de riscos e agravosà saúdeindividual e coletiva, atenção à saúde com equidade e integralidade, expansão, intervenção einovação tecnológica em saúde.

Essas "linhas de ação" foram substratos à composição dos programas de governo constantes do capítulo da Saúde do plano plurianual (PPA 2008-2011) eà elaboração da agenda estratégica da saúde para os anos de 2007 e 2008, processos desenvolvidos com intensa participação institucional: seiscentos servidores do nível central edas Diretorias Regionais deSaúde(DIRES), mais de trinta reuniões/oficinas detrabalho, sete grandes encontros para validação dos produtos parciais do processo de planejamento envolvendo todos os níveis decisórios da instituição. Adotou-se, também, como referência normativa para essa construção, as diretrizes do Pacto pela Saúde $^{32}$ e do Sistema de Planejamento do SUS (PlanejaSUS) ${ }^{8}$.

Entre abril e julho de 2007, a SESAB participou também de uma série de encontros com re presentantes da sociedade civil organizada em 26 regiões do Estado, denominadas territórios de identidade ${ }^{33}$, com a finalidade de levantar as demandas que subsidiariam a elaboração do PPA e, a posteriori, subsidiaram a análise situacional.

Ainda no contexto deimplementação das"Iinhas de ação" da gestão sob a coordenação da Diretoria de Programação e Desenvolvimento da Gestão Regional, da Superintendência de Gestão da Regulação da Atenção à Saúde (DIPRO/SU REGS), elaborou-se um novo Plano Diretor de Regionalização (PDR) 34 , aprovado pela Comissão Intergestores Bipartite (CIB). 0 estado passou a contar com nove macrorregiões de saúde, territórios onde se promoveu a organização das microrregiões de saúde, espaços de implementação de redes assistenciais em áreas estratégicas.

Enquanto a ASPLAN (Assessoria de Planejamento) conduziu em conjunto com o Fundo Estadual de Saúde da Bahia (FESBA) o processo de elaboração do PPA 2008-2011, a DIPRO conduziu o processo de revisão do PDR. Esses dois instrumentos, normativo e organizacional, constituíram-se em dois grandes balizadores da gestão na construção do Plano Estadual de Saúde.
Terceiro movimento:

a análise da situação de saúde (ASIS)

O processo de elaboração do PES 2008-2011 teve a "análise de situação de saúde" ${ }^{35}$ como componente prioritária à elaboração de planos de saúde nos diversos níveis de gestão do SUS. Em função disso, a Diretoria de Informação em Saúde(DIS) tratou de atualizar e sistematizar informações relativas ao perfil epidemiológico da população baiana, enquanto a Superintendência de Gestão do Sistema e Regulação da Aten ção à Saúde (SUREGS) sistematizou a descrição da organização dos serviços de saúde no estado. Essas duas análises foram construídas levando-se em conta a subdivisão do território do estado nas nove macrorregiões de saúde definidas no PDR.

O perfil epidemiológico foi construído com base na análise dos indicadores de morbimortalidade constantes nos sistemas de informação de base nacional. Evidencia-se o envel hecimento da população baiana e a morte prematura de jovens, bem como se revela a transição epidemiológica que vem ocorrendo no estado, com a coexistência de doenças da modernidade, da pobreza e doenças reemergentes. A desagregação das informações epidemiológicas por macrorregião de saúde foi complementada pela análise da gestão do sistema eorganização dos serviços desaúde de forma regionalizada, evidenciando-se a concentração deserviços em número e especialidade, particularmenteno município de Sal vador, para onde convergem usuários de todos os municípios do estado.

Quarto movimento: a consolidação

das demandas e recomendações

das conferências de saúde

Ainda em 2007, realizou-se a 7 ạ Conferência Estadual de Saúde, cujo tema central "Saúde e Qualidade deVida - Políticas de Estado e D esenvolvimento" foi previamente discutido nas 393 Conferências M unicipais de Saúde, cujos relatórios possibilitaram a formação de um banco de dados com as mais de cinco mil propostas da sociedade civil ${ }^{36}$, que por sua vez foram consideradas na realização dos movimentos seguintes.

Em seguimento ao processo de realização das conferências de saúde, a ASPLAN organizou as demandas e recomendações emanadas desses fóruns, segundo as linhas de ação estabelecidas no planejamento da SESAB. Cada demanda/recomendação foi inserida em uma área temática de planejamento $(n=31)$, desenvolvida no con- 
texto de cada linha de ação e macrorregi ão, considerando o desenho do novo PDR. Esse movimento configurou-se numa importante estraté gia de escuta para orientar a elaboração do PES 2008-2011, sendo balizador dos problemas identificados na análise de situação de saúde e subsídio para as priorizações decorrentes das oficinas de construção do plano.

Quinto movimento: as oficinas integradas de priorização de problemas de estado de saúde da população e sistema de serviços de saúde

A SESAB avançou, em 2008, para a real ização de um conjunto de "oficinas integradas macrorregionais", com o propósito de reunir e articular os diversos elementos trabalhados até aquele momento. N essa perspectiva, desenvolveram-se também processos de revisão das políticas setoriais que se constituíram nas referências normativas do processo, consolidadas posteriormente no capítulo do plano que trata das políticas e diretrizes.

Os objetivos dessas oficinas foram apresentar as análises de saúde desenvolvidas por técnicos do nível central (do estado de saúde da população e da organização de serviços de saúde), chancelar as informações com os demais atores responsáveis pela formulação de políticas de saúde (conselheiros, universidades, COSEM S, gestores municipais etécnicos do nível central eDIRES) e validar as análises obtidas a partir de bancos de dados das necessidades sentidas em cada macrorregião de saúde no Estado da Bahia.

Sexto movimento:

a avaliação de desempenho da SESAB

e fortalecimento das Funções Essenciais de Saúde Pública (FESP/SUS)

Paral el amente à realização da ASIS edivulgação desta nas oficinas integradas, desencadeouse, ao final de 2007, um processo de avaliação de desempenho institucional que incluiu a análise do grau de cumprimento de metas acordadas na agenda estratégica da Saúde em 2007, bem como contemplou a construção de um conjunto de indicadores que foram avaliados em cada setor ${ }^{37}$.

Com base nessa avaliação e na discussão ampliada sobre os resultados obtidos com a implementação das ações da agenda em 2007, foi elaborada a agenda estratégica da saúde para 2008, cujo formato representa um avanço metodológico em relação à anterior. Os objetivos es- tratégicos foram tomados como "compromissos" de cada órgão e traduzidos em "resultados" a serem alcançados em um prazo definido.

Dando seguimento ao processo de avaliação de institucional, optou-se por adotar a proposta de fortalecimento da SESAB elaborada como parte da avaliação das "Funções Essenciais de Saúde Pública", proposta pela Organização PanAmericana de Saúde (OPAS/OMS) e adaptada para a Gestão Estadual do SUS pelo Conselho N acional de Secretários Estaduais de Saúde (CONASS) ${ }^{38}$, que se desenvolveu em duas etapas conduzidas pela SESAB em articulação com o M inistério da Saúde (MS), por meio do PlanejaSUS.

Esse processo constituiu-se em um marco para o processo avaliativo do SUS no estado, pelo seu caráter coletivo e participativo, aproximando eintegrando as instâncias corresponsáveispela gestão do SUS estadual: os dirigentes da SESAB, o Conselho Estadual de Saúde (CES), o ConseIho de Secretários M unicipais de Saúde da Bahia (COSEM S/BA), o M inistério da Saúde (MS), os dirigentes de hospitais universitários, as universidades públicas e privadas e Secretarias de Governo da Bahia.

A realização da oficina de autoavaliação no estado contou com a garantia das condições propostas pela coordenação nacional para a realização da oficina de modo voluntário pelo Secretário de Saúde, por meio de ofício ao CONASS, incluindo a logística do evento e a indicação da coordenação estadual da autoavaliação.

A Bahia foi o décimo primeiro estado a realizar a autoavaliação das FESP/SUS. 0 processo envolveu três grupos de trabalho, num total de 91 pessoas entre participantes (75) e observadores (16). Entre os partici pantes, contou-se com a presença daqueles externos à Secretaria Estadual, entre eles, dez professores de universidades, seis representantes do Consel ho Estadual deSaúde, quatro representantes demunicípios, além de representantes de outras secretarias de governo, como SEPLAN e Casa Civil. Entre os observadores, contou-se com a presença de dois representantes do M inistério da Saúde, Secretaria de Gestão Participativa e do Departamento de Apoio à Descentralização, um técnico da OPAS/OMS, além de técnicos da própria SESAB, em especial, do setor de planejamento (8) ${ }^{39}$.

A Oficina de Fortalecimento das FESP do SUS-Bahia, segundo momento do processo de fortalecimento das FESP no estado, teve como propósito a construção de uma agenda de compromissos a partir do resultado alcançado pela avaliação, visando fortalecer as macrofunções da 
gestão estadual, considerando o referencial das FESP. Nela, foram discutidos os pontos fracos identificados na avaliação e elaborado um plano de ação para melhorar o desempenho do sistema público de saúde no estado, considerando o contexto e as iniciativas de implementação de processos que visam ao desenvolvimento institucional do SUS-Bahia ${ }^{40}$.

Sétimo movimento:

a construção dos "compromissos"

do plano - módulos operacionais

A elaboração de módulos operações-problemas, coerentemente com o referencial do plane jamento estratégico-situacional ${ }^{26,27}$ adotado, tomou como ponto de partida a identificação dos problemas, demandas e oportunidades sob responsabilidade direta dos gestores que ocupam postos estratégicos no sistema/instituição (Figura 1). Organizaram-se, portanto, oficinas por "linha de ação", conduzidas pelo GT de Planejamento, nas quais foram definidos objetivos específicos que detal ham os compromissos de gestão, bem como foram redesenhadas as ações estratégicas para seu cumprimento. I dentificaramse também os resultados esperados para cada ação, indicados recursos orçamentários e a corresponsabilidade dos diversos setores da instituição por cada uma delas, mantendo-se a busca de articulação entre o PPA e o PES, sendo construídos treze módulos operacionais/compromissos, quais sejam: (1) gestão estratégica, participativa e efetiva do SUS, (2) regionalização viva e solidária, (3) regulação, controle e avaliação do acesso da população aos serviços de saúde do SUS, (4) política estadual de gestão do trabalho e da educação permanente em saúde, com ênfase na desprecarização das relações de trabalho, (5) vigilância da saúde com integração das práticas nas esferas estadual e municipais do SU S-Bahia, (6) atenção básica com inclusão social e equidade - "Saúde da família de todos nós", (7) atenção especial izada regionalizada, resolutiva e qualificada, com base nas linhas de cuidado e considerando as necessidades de saúde da população, (8) atenção integral à saúde de populações de maior vulnerabilidade social e em situações especiais de agravo, com vistas à redução de iniquidades, (9) assistência farmacêutica para todos os baianos, (10) assistência hematológica e hemoterápica descentralizada e regionalizada, (11) expansão e melhoria da infraestrutura administrativa e dos serviços de saúde do SUS-Bahia, (12) expansão da base científica e tecnológica do
SUS-Bahia e (13) políticas transversais para a promoção da saúde, segurança alimentar e proteção da sociedade.

\section{O documento final do Plano Estadual de Saúde da Bahia 2008-2011}

O PES 2008-2011, considerado um dos marcos normativos do SUS-Bahia, busca consolidar o conjunto das políticas públicas da área de saúde. Está ancorado, em âmbito estadual, no projeto estratégico do governo ${ }^{41}$ para a área de saúde, definida como prioritária; em âmbito federal, na Política Nacional de Saúde, explicitada por meio do Plano Nacional de Saúde ${ }^{42}$ e de políticas específicas como o Pacto pela Saúde ${ }^{32}$ eo PlanejaSU S8; busca refletir ainda as prioridades estabelecidas no programa "M ais Saúde" do governo federa ${ }^{43}$, preocupa-se com as prioridades estabelecidas na Agenda de Saúde das Américas ${ }^{44}$; coaduna com a proposta das Funções Essenciais de Saúde Públi$\mathrm{ca}(\mathrm{FESP})^{38}$ e prioriza intervenções para garantir 0 alcance das metas estabelecidas nos objetivos do milênio na área de saúde ${ }^{45}$.

As diretrizes explicitadas no documento constituem elementos da agenda governamental para a construção do SUS no estado e traduzem a direcionalidade estratégica que se busca imprimir ao sistema: (1) regionalização solidária da atenção à saúde, (2) gestão do trabalho e da educação permanente em saúde, (3) intersetorialidade e transversalidade nas ações, (4) valorização do trabalhador do SUS, (5) comunicação e diálogo com os parceiros e sociedade (6) resolutividade e satisfação do usuário do SUS.

A estrutura do PES 208-2011 constitui-seem um conjunto de capítulos que contemplam os diversos subprodutos do processo de elaboração, como segue:

- Política de Saúde na Bahia: princípios e diretrizes - apresentando o escopo político-ideológico, princípios, diretrizes emarcos conceituais que sustentam as intervenções de governo e organizam o cotidiano do componente estadual da gestão do SUS em articulação com o M inistério da Saúde e se configuram numa possibilidade de pactuação com os gestores municipais;

- Consolidado da análise situacional por macrorregião desaúde- contendo um recortedo perfil epidemiológico do Estado da Bahia, uma breve discussão acerca da estrutura e organização dos serviços de saúdeeuma síntese dos problemas prioritários de estado de saúde da população e do sistema desaúde, ancorados na priorização de pro- 
blemas realizada nas oficinas integradas macrorregionais e na análise das demandas e recomendações do PPA-Participativo e das conferências municipais eda conferência estadual de saúde;

- M atriz estratégica do Plano Estadual deSaúde - apresentando uma síntese esquemática da organização das linhas de ação, compromissos, objetivos específicos, explicitando, ainda, a visão de futuro e o macroobjetivo do plano e sua necessária articulação com os princípios e diretrizes explicitadas na política estadual de saúde;

- Linhas de ação - apresentando as sete "linhas de ação" norteadoras da gestão do SU SBahia, construídas desde a elaboração do PPA 2008-2011, a partir da análise e consolidação das demandas e necessidades explicitadas no movimento do PPA-Participativo. Revisa o conteúdo dessas "linhas de ação", ampliando-as com a inclusão dos resultados da análise da situação de saúde, que aponta as prioridades de intervenção sobre o estado de saúde da população e sobre a organização do sistema em todo o estado;

- Viabilidadefinanceiro-orçamentária do Plano Estadual de Saúde: conexão com o PPA 2008/ 2011 - demonstrando em que medida os programas do PPA 2008-2011 se articulam com os compromissos explicitados no plano, isto é, onde estão alocados os recursos orçamentários previstos para a execução das ações estratégicas propostas para 0 alcance das metas e resultados esperados no período.

\section{Análise do processo de construção} do PES 2008-2011

A análise do processo de construção do PES 20082011 pode ser realizada de uma dupla perspectiva: (1) considerando a opção teórico-conceitual pelo enfoque estratégico-situacional, explicitada anteriormente, cabe analisar a coerência interna dos distintos movimentos e dos produtos parciais elaborados a cada passo, com os momentos do planejamento estratégico-situacional (PES); (2) considerando a posição ocupada por um dos autores no processo, enquanto dirigente responsável pela organização e condução do planejamento, cabe discutir os avanços e desafios a serem enfrentados com vistas à consolidação das práticas de planejamento na instituição.

N esse sentido, este item traz, inicialmente, a análise do processo de elaboração do PES e do produto resultante, para, em seguida, apresentar uma apreciação dos aspectos positivos e ne gativos da experiência ainda em processo.
Articulação dos tempos e movimentos

Buscando articular o conjunto desses processos com vistas à análise compreensão abrangente da sua complexidade, foi construída uma "linha do tempo" (Figura 2) - diagrama que busca sintetizar os processos e produtos desenvolvidos ao longo do período de construção do PES 2008-2011. Esse diagrama toma como base teórica os momentos do planejamento estratégicosituacional, que necessariamentenão tem começo nem fim definidos, quais sejam: o momento explicativo, o momento normativo, o momento estratégico e o momento tático-operacional ${ }^{24}$.

0 momento explicativo se estende desde 0 processo de elaboração do diagnóstico da transição, em novembro de 2006, até a finalização da análise de situação de saúde que subsidiou a construção do PES 2008-2011, em novembro de 2008. Pode-se afirmar, com base no referencial adotado ${ }^{28,29}$, que esse momento se caracterizou pela construção de uma visão compartilhada na instituição sobre a realidade dos problemas de saúde da população e do sistema estadual de saúde, bem como pela intensa reflexão acerca da cultura organizacional vigente na SESAB e as possibilidades de mudança.

Como produtos parciais, podem-se identificar a definição dos macroproblemas da gestão do SUS no estado, a sistematização das demandas oriundas das conferências municipais de saúde e a definição do escopo dos problemas desaúde explicitados por meio da análise situacional elaborada ao longo do período. Por fim, pode ser incluído também o movimento da autoavaliação das FESP, cujo relatório final subsidiou o processo de priorização, por parte do Colegiado de Gestão da SESAB, dos principais problemas a serem enfrentados no âmbito da gestão do sistema estadual de saúde.

0 momento normativo revela-se na construção do "programa direcional" ${ }^{24}$ e, a partir daí, todos os documentos dele decorrentes. Estemomento pode ser visto como aquele que contribui para a consolidação do pensamento sistêmico na instituição. Pode-se dizer que a concretização do referido programa direcional se deu através da definição da "matriz estratégica do SUS-Bahia", que explicita, por meio das linhas de ação, a direcionalidade do projeto de governo na área de saúde. Essa matriz direcionou a elaboração do Plano plurianual de governo, o PPA 2008-2011, as leis de diretrizes orçamentárias e leis orçamentárias anuais, bem como as agendas estratégicas para os anos de 2007, 2008, 2009 e2010. 0 docu- 


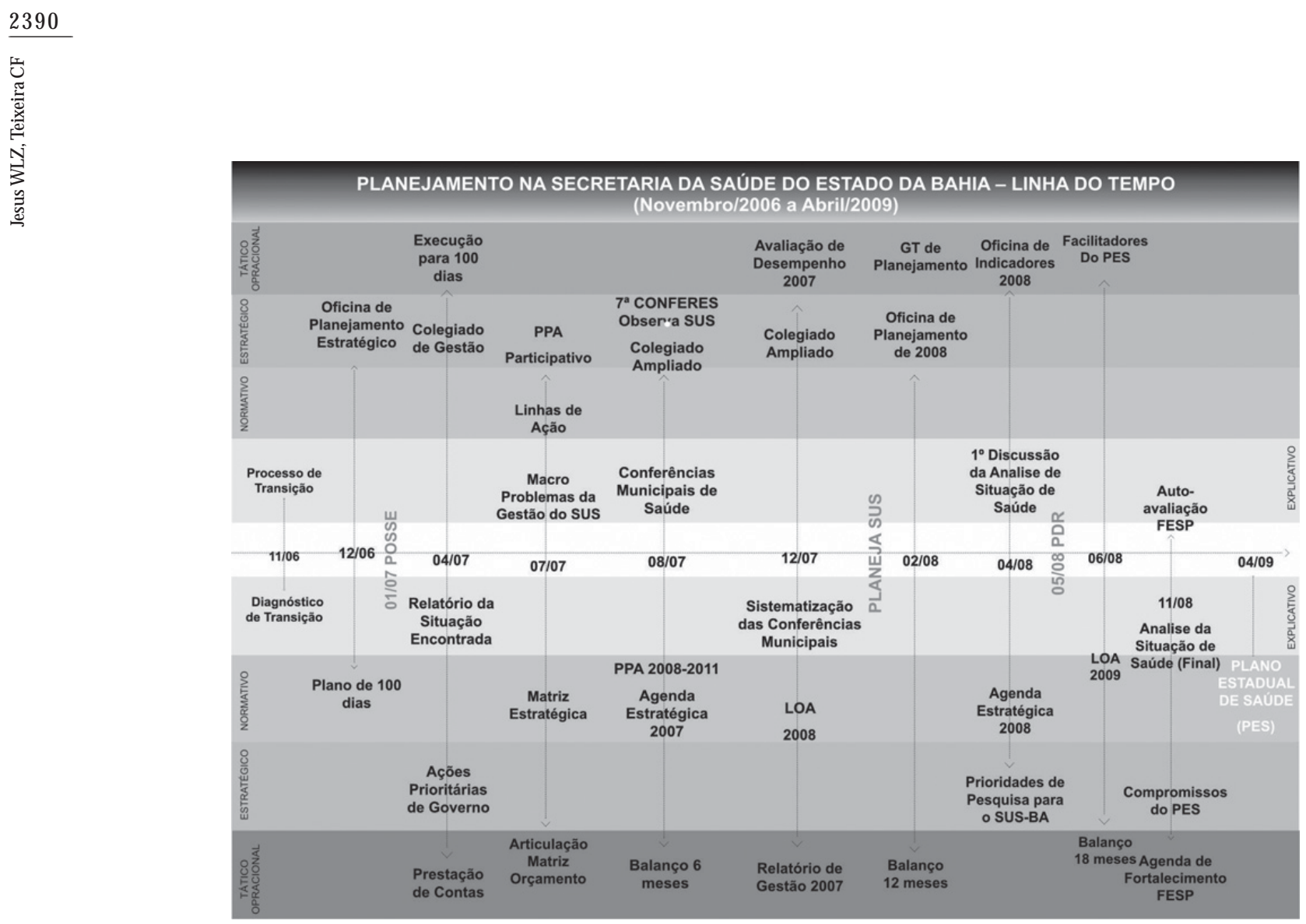

Figura 2. Linha do tempo do processo de elaboração do PES/Bahia 2008-2011.

mento que consolida o momento normativo éo próprio Plano Estadual de Saúde, cuja construção, no caso, se deu de modo estritamente modular como propõe Matus.

0 momento estratégico se explicita no processo através das decisões tomadas pela gestão colegiada e que acabam por conformar os caminhos da implementação das proposições do Plano Estadual de Saúde, um momento em que se pode verificar a essência da aprendizagem em equipe e do diálogo por meio do exercício da liderança e negociação coletiva. N esse momento, pode-se destacar a atuação do Colegiado de Gestão da SESAB e o colegiado ampliado da instituição, que definiram o escopo das ações prioritárias de governo e os compromissos da gestão para o período, identificando os pontos críticos de intervenção, as contingências e, quando necessário, redesenhando a agenda político-gerencial. Também é parte desse processo a atuação dos gestores junto à Comissão Intergestores Bipartite (CIB) eao Conselho Estadual de Saúde(CES), estabelecendo os pactos necessários para 0 desenvolvimento das políticas contidas no plano, bem como a articulação da SESAB com a sociedade civil para definição de prioridades no PPAPartici pativo enas conferências de saúdee com a comunidade acadêmica na definição das priori- dades de pesquisa para o SUS na Bahia segundo as linhas de ação definidas na matriz estratégica.

0 momento tático-operacional, como o próprio termo sugere, traz consigo o fazer cotidiano da instituição, cuja materialização se dá desde o momento da execução do Plano de 100 dias, que marca o início da gestão a partir de janeiro de 2007, atéa definição do Plano de Fortalecimento da Gestão da SESAB (produto das FESP) incorporada à agenda estratégica da saúde (20092010). Requer da instituição o exercício derevisitar sua cultura e, por meio da aprendizagem coletiva e do diálogo, estabelecer os pactos necessários para avançar nos propósitos. 0 ator fundamental desse momento foi o GT de Planejamento eAvaliação e o trabal ho implicou o redesenho contínuo das intervenções ao longo do período. Como parte dos movimentos desenvolvidos no âmbito do momento tático-operacional, está toda a sistemática desenvolvida para "prestar contas" da execução do projeto de governo, bem como os movimentos quese desenvolveram para a conformação do próprio documento do plano, como, por exemplo, o trabal ho conjunto entre a SESAB e o ISC-UFBA para construir as oficinas integradas para seleção e priorização dos problemas de saúde que subsidiaram a consolidação do PES 2008-2011. 
Facilidades e dificuldades, avanços e desafios

Todos os movimentos até então explicitados implicam, portanto, em construir a mudança e consolidar o compromisso dos sujeitos participantes que planejam, articulam e integram a sociedade em busca da transformação do sistema estadual de saúde, e se propõem a orientar as ações da gestão nas formulações e execuções das políticas e práticas de saúde.

Destacam-se, como facilidades, a vontade política do gestor estadual (governador e secretário da saúde) e dos diversos atores políticos em construir uma proposta participativa de planejamento para o sistema de saúde, a definição de um eixo condutor pautado no enfoque estratégico-situacional para o desencadeamento dos movimentos adotados no processo de construção do Plano Estadual deSaúdeeo impulso dado pela institucionalização do PlanejaSU S a esse processo, na medida em que viabilizou a capacitação de pessoal e o estabelecimento de parcerias interinstitucionais que contemplam a execução de consultorias e o desenvolvimento de estudos e pesquisas com a finalidade de subsidiar 0 aperfeiçoamento da prática de planejamento em saúde no estado.

No tocante às dificuldades, podem-se identificar a complexidade de processos participativos na prática, o tempo político de uma gestão, restrito a quatro anos, o que reduz a capacidade de implementação das propostas construídas, cujos resultados almejados vão para além do período de vigência do plano, a limitação dos recursos para intervir sobre a totalidade dos problemas, as dificuldades de entendimento quanto às diferentes concepções sobre planejamento e sua apreensão pel os diversos atores institucionais ea convivência entreo "velho" e o "novo" no cenário político-institucional - velhas concepções e novas roupagens.

Como avanços, destacam-seo reconhecimento da importância da participação dos diversos atores sociais na formulação das políticas públicas em saúde, a adoção do enfoque sobre problemas, o reconhecimento de que o planejamento só se completa na "ação", configurando-se como movimento processual de construção, capaz de impulsionar e redirecionar a elaboração do plano a todo o momento, a articulação entre elementos de planejamento e avaliação e o reconhecimento pela própria instituição da importância do processo para sua reorganização interna, tendo em vista que a direcionalidade dada pelas "linhas de ação" produziu rearranjos institucionais formais e informais, além da identificação dos limites da própria instituição para agir, produzindo, assim, uma ambiência de aprendizagem organizacional que aproxima a SESAB da perspectiva da "learning organization" defendida por Senge ${ }^{29}$.

Por fim, quanto aos desafios, cabe destacar queo processo deaprendizagem institucional demanda da instituição e daqueles que a compõem novas posturas. Apreende-se, dessa experiência, que a implementação de processos participativos de construção de políticas requer dos sujeitos envolvidos uma maior conscientização quanto à necessidade de escuta, perseverança, respeito ao pensamento e à construção coletiva, o que, em última análise, nos permiteafirmar que esses processos respondem aos ideários estabelecidos no próprio SUS, nos quais o usuário-cidadão deve ser o centro das políticas públicas.

\section{Consideraçõesfinais}

0 processo de planejamento para a elaboração do PES (2008-2011) na Secretaria da Saúde do Estado da Bahia se deu por meio da combinação de diversos tempos e movimentos. 0 referencial teórico adotado, na perspectiva do enfoque situacional, permitiu maior flexibilidade metodológica, proporcionando aos condutores do processo articular diferentes métodos e construir, de forma sistemática, o plano com intrínseca relação ao plano plurianual (PPA) e possibilitou coordenar na sequência a elaboração de uma agenda estratégica para os anos de 2009 e 2010.

Desde a proposta do planejamento integrado até a construção dos módulos operacionais, ocorreu, de certo modo, um amadurecimento na equipedirigente, na medida em que exercitou a construção de consensos e pactos em torno de compromissos, resultados esperados, ações estratégicas e metas correspondentes em cada setor da instituição. É possível supor que isso tenha implicado uma maior responsabilização político-gerencial dos sujeitos envolvidos e desencadeado também movimentos de aprendizagem institucional.

A descrição e análise desse processo revelam a potência do planejamento, numa perspectiva situacional, e demonstram que, mesmo diante da pluralidade e multiplicidade de abordagens existente na área, é possível conduzir as instituiçõesa movimentos deconstrução coletiva deuma "visão de futuro" que contribua para a coesão 
interna entre a equipe dirigente, quadros técnicos e trabalhadores que atuam no cotidiano dos serviços. N essa perspectiva, a articulação do momento de explicação da situação de saúde, com a elaboração dos contornos da situaçãoobjetivo pretendida no horizonte temporal do plano, bem como com a definição de estratégias

\section{Colaboradores}

WLA Jesus foi responsável pela concepção, delineamento, análise, interpretação e redação do artigo. CF Teixeira também foi responsável pelo delineamento, análisee interpretação, além deter feito revisão crítica e aprovação da versão a ser publicada.

\section{Agradecimentos}

Este artigo é fruto da sistematização de um processo de trabalho coletivo que envolveu um conjunto de sujeitos que participaram direta e indiretamente do processo de planejamento da SESAB no período 2007-2009, especialmente o Colegiado de Gestão da SESAB, a equipe da Assessoria dePlanejamento/Coordenação de Projetos Especiais e o Grupo Técnico de Planejamento e Avaliação em Saúde desta instituição. Também há que se considerar a participação dos residentes da UFBA e da UNEB, das diversas áreas, que ao longo do período participaram das iniciativas que deram origem a esse artigo. a serem desenvolvidas para a consecução das mudanças necessárias na organização e gestão do sistema público de saúde, identifica o "plano" como uma aposta na qualificação das ações de um governo ${ }^{24}$, comprometido com a consolidação dos direitos de cidadania e com a consolidação do SUS.

\section{Referências}

1. Rivera FJU, Artmann E. Planejamento e gestão em saúde: flexibilidade metodológica e agir comunicativo. Cien Saude Colet 1999; 4(2):355-365.

2. M erhy EE. Planejamento como tecnologia de gestão: tendências e debates em saúde no Brasil. In: Gallo $E$, organizador. Razão e planejamento: reflexões sobre política, estratégia e liberdade. São Paulo: Hucitec; Rio de Janeiro: Abrasco; 1995. p. 117-148.

3. Schraiber LB, Peduzzi M, Sala A, Vemes MIB, Castanhera ERL, Kon R. Planejamento, gestão e avaliação em saúde: identificando problemas. Cien Saude Colet 1999; 4(2):221-242.

4. Kalil MEX, Paim JS. Planejamento de saúde na Bahia: impasses e perspectivas. Rev. baiana saúde pública 1986; 13(1/3):47-67.

5. Paim JS. Gestão do SUDS na Bahia. Cad Saude Publica 1989; 5(4):365-375.

6. Paim JS. Aspectos críticos da institucionalização da planificação em organizações públicas de saúde. In: Paim JS. Saúde: política e reforma sanitária. Salvador: Cooptec/ISC; 2002.

7. Bahia. Secretaria da Saúde. Relatório da situação encontrada: planejamento na SESAB (portfólio de documentos institucionais). Salvador: SESAB; 2007. [M imeo]

8. Brasil. M inistério da Saúde. Sistema de planejamento do SUS: organização e funcionamento. Brasília: Ministério da Saúde; 2006.

9. Levcovitz E, Baptista TWF, U chôa SAC, Nesploli G, Mariani M. Produção do conhecimento em política, planejamento e gestão em saúde e políticas de saúde no Brasil (1974-2000). Brasília: OPAS; 2003.

10. Paim JS, Teixeira CF. Política, Planejamento e gestão em saúde: balanço do estado da arte. Rev. Saude Publica 2006; 40(N Esp):73-78.

11. Coelho TCB. 0 processo de gestão em uma organização hipercomplexa do Sistema Ú nico de Saúde [tese]. Salvador (BA): ISC/UFBA; 2002.

12. Lotufo $M, M$ iranda $A$. Sistemas de gestão e práticas de gestão governamental em secretarias estaduais de saúde. RAP 2007; 41(6):1143-1163.

13. Vilasbôas ALQ. Prática de planejamento e implementação de políticas de saúde no âmbito municipal [tese]. Salvador (BA): ISC/UFBA; 2006.

14. Rocha AARM. O planejamento no cotidiano de uma instituição hipercomplexa: 0 caso da SES/ Sergipe [tese]. Salvador (BA): ISC/U FBA; 2008. 
15. Cerqueira SCC. 0 processo de incorporação do Plano M unicipal de Saúde como tecnologia de gestão: 0 caso da Secretaria M unicipal de Saúde de Salvador [dissertação]. Salvador (BA): ISC/UFBA; 2009.

16. Yin RK. Estudo de caso: planejamento e métodos. 3a ed. Porto Alegre: Bookman; 2005.

17. Bahia. Secretaria da Saúde. Plano Estadual de Saúde 2008-2011. Salvador: SESAB; 2009.

18. Bahia. Secretaria da Saúde. Plano Plurianual de Governo, PPA 2008-2011. Salvador: SESAB; 2007.

19. Bahia. Secretaria da Saúde. Agenda Estratégica da Saúde 2007. Salvador: SESAB; 2007.

20. Bahia. Secretaria da Saúde. Agenda Estratégica da Saúde 2008. Salvador: SESAB; 2008.

21. Bahia. Secretaria da Saúde. Agenda Estratégica da Saúde 2009/2010. Salvador: SESAB; 2009.

22. Testa M. Pensamento estratégico e lógica da programação. São Paulo: Hucitec; 1995.

23. Testa M. Análisis de instituciones hipercomplejas. In: Merhy EE, Onocko RT, organizadores. Agir em saúde: um desafio para o público. 2ª ed. São Paulo: Hucitec; 2002. p.17-70.

24. Matus $C$. Planejamento e programação em saúde: um enfoque estratégico. São Paulo: Cortez; 1989.

25. Matus C. Política, planificação e governo. $2^{\mathrm{a}}$ ed. Brasília: IPEA; 1993. p. 297-554.

26. Matus C. Política y plan. Caracas: Iveplan; 1982.

27. Teixeira CF. Planejamento municipal em saúde. Salvador: ISC/UFBA; 2001.

28. Rivera FJU, Artmann E. Planejamento e gestão em saúde: flexibilidade metodológica e agir comunicativo. In: Rivera FJU, organizador. Análise estraté gica em saúde e gestão pela escuta. Rio de Janeiro: Fiocruz; 2003. p. 17-33.

29. Rivera FJU. Planejamento e programação em saúde: um enfoque estratégico. 2aㅡ ed. São Paulo: Cortez; 1992.

30. Senge PM . A quinta disciplina: arte e prática da organização que aprende. 24a ed. Rio de Janeiro: BestseIler, 2008.

31. Moreira MR, Escorel S. Conselhos M unicipais de Saúde do Brasil: um debate sobre a democratização da política de saúde nos vinte anos do SUS. Cien Saude Colet [periódico na Internet]. 2009 [acessado 2010 mar 15];14(3):[cerca de 12 p.]. Disponível em: http://www.scielo.br/pdf/csc/v14n3/15.pdf

32. Brasil. M inistério da Saúde. Pacto Pela Saúde. Brasília: Ministério da Saúde; 2006.
33. Bahia. Secretaria de Planejamento. Territórios de Identidade da Bahia. Salvador: SEPLAN; 2007.

34. Bahia. Secretaria da Saúde. Plano Diretor de Regionalização - PDR. Salvador: SESAB; 2007.

35. Castellanos PL. Epidemiologia, saúde pública, situação de saúde e condições de vida: considerações conceituais. In: Barata R, organizadora. Condições de vida e situação de saúde. Rio de Janeiro: Abrasco; 1997. p. 31-75.

36. Bahia. Secretaria da Saúde. Relatório Anual de Gestão 2007. Salvador: SESAB; 2008.

37. Bahia. Secretaria da Saúde. Relatório de Avaliação de Desempenho Institucional da SESAB 2007. Salvador: SESAB; 2008.

38. Brasil. Conselho Nacional de Secretários de Saúde. A gestão da saúde nos estados: avaliação e fortalecimento das funções essenciais. Brasília: CONASS/ OPAS; 2007.

39. Bahia. Secretaria da Saúde. Relatório de Avaliação de Desempenho das Funções Essenciais de Saúde Pública. Salvador: SESAB; 2008.

40. Bahia. Secretaria da Saúde. Relatório de Avaliação da O ficina de Fortalecimento da Gestão Estadual do SUS nas Funções Essenciais de Saúde Pública. Salvador: SESAB; 2009.

41. Bahia. Secretaria da Saúde. Planejamento Estratégico Setorial da Saúde. Salvador: SESAB; 2007. [M imeo]

42. Brasil. M inistério da Saúde. Plano Nacional de Saúde 2003-2007. Brasília: Ministério da Saúde; 2007.

43. Brasil. M inistério da Saúde. Programa Mais Saúde PAC Saúde. Brasília: Ministério da Saúde; 2007.

44. Organização Pan-Americana de Saúde. Organização Mundial de Saúde. Agenda de Saúde para as Américas 2008-2017. Panamá: OPAS/OM S; 2007.

45. Brasil. O bjetivos de desenvolvimento do milênio: relatório nacional de acompanhamento. Brasília: IPEA; 2007.

Artigo apresentado em 26/04/2010

Aprovado em 25/05/2010

Versão final apresentada em 10/06/2010 